\title{
Properties of ${ }^{12} \mathrm{C}$ resonances determined from the ${ }^{10} \mathrm{~B}\left({ }^{3} \mathrm{He}, p \alpha \alpha \alpha\right)$ and ${ }^{11} \mathrm{~B}\left({ }^{3} \mathrm{He}, d \alpha \alpha \alpha\right)$ reactions studied in complete kinematics
}

\author{
M. Alcorta, ${ }^{1,2,{ }^{*}}$ M. J. G. Borge,${ }^{1}$ M. Cubero,${ }^{1}$ C. Aa. Diget,${ }^{3, \dagger}$ R. Domínguez-Reyes,,${ }^{1, \dagger}$ L. M. Fraile, ${ }^{4}$ B. R. Fulton, ${ }^{5}$ \\ H. O. U. Fynbo, ${ }^{3}$ D. Galaviz, ${ }^{1,}{ }^{\S}$ S. Hyldegaard, ${ }^{3}$ H. Jeppesen, ${ }^{6}$ B. Jonson, ${ }^{7}$ O. S. Kirsebom, ${ }^{3, \|}$ M. Madurga, ${ }^{1, \uparrow}$ A. Maira, ${ }^{1}$ \\ A. Muñoz-Martín, ${ }^{8}$ T. Nilsson, ${ }^{7}$ G. Nyman, ${ }^{7}$ D. Obradors, ${ }^{7}$ A. Perea, ${ }^{1}$ K. Riisager, ${ }^{3}$ O. Tengblad, ${ }^{1}$ and M. Turrion ${ }^{1, * *}$ \\ ${ }^{1}$ Instituto de Estructura de la Materia, CSIC, Madrid E-28006, Spain \\ ${ }^{2}$ Physics Division, Argonne National Laboratory, Argonne, Illinois 60439, USA \\ ${ }^{3}$ Department of Physics and Astronomy, Aarhus University, DK-8000 Aarhus C, Denmark \\ ${ }^{4}$ Grupo de Física Nuclear, Universidad Complutense, E-28040 Madrid, Spain \\ ${ }^{5}$ Department of Physics, University of York Heslington, UK \\ ${ }^{6} \mathrm{PH}$ Department, CERN, CH-1211 Geneve, Switzerland \\ ${ }^{7}$ Fundamental Physics, Chalmers University of Technology, Gothenburg, Sweden \\ ${ }^{8}$ CMAM, Universidad Autonoma de Madrid, Cantoblanco, Spain
}

(Received 10 August 2012; published 10 December 2012)

\begin{abstract}
We have used the ${ }^{10} \mathrm{~B}\left({ }^{3} \mathrm{He}, p \alpha \alpha \alpha\right)$ reaction at $4.9 \mathrm{MeV}$ and the ${ }^{11} \mathrm{~B}\left({ }^{3} \mathrm{He}, d \alpha \alpha \alpha\right)$ reaction at $8.5 \mathrm{MeV}$ to determine the energy, width, and decay mechanism of resonances in ${ }^{12} \mathrm{C}$ above the triple- $\alpha$ threshold up to $21 \mathrm{MeV}$ in excitation energy. Comparison with various models has allowed us to estimate the degree of clustering of the states studied.
\end{abstract}

DOI: 10.1103/PhysRevC.86.064306

PACS number(s): 27.20.+n, 21.45.-v, 21.60.Gx, 25.55.-e

\section{INTRODUCTION}

Although the nuclear structure of ${ }^{12} \mathrm{C}$ has been under scrutiny since the beginning of nuclear physics, important questions remain unanswered. The main interest in this nucleus is also the main cause of difficulty: the mixture of two types of structures which have either single nucleons or $\alpha$ particles as effective degrees of freedom. In the physical states of the ${ }^{12} \mathrm{C}$ nucleus, these types of motion are mixed, which makes the use of models built on one or the other inadequate. For example, shell-model calculations fail to reproduce the second excited state, which is the famous $0^{+}$state at $7.65 \mathrm{MeV}$, due to strong $\alpha$-cluster correlations in this state [1]. ${ }^{12} \mathrm{C}$ is the focus of several $a b$ initio theoretical frameworks such as Green's function and variational Monte Carlo calculations [2], and nuclear lattice calculations $[3,4]$. However, these approaches are still far from being able to provide a full understanding of the interplay of the two different structures. Presently, the most successful approaches for understanding the interplay between single-nucleon and cluster correlations are antisymmetrized

\footnotetext{
*malcorta@anl.gov

†Present address: Department of Physics, University of York, Heslington, UK.

${ }^{\ddagger}$ Present address: Departamento de Física, Universidad Carlos III de Madrid, Avd. Universidad 30, 28911 Leganés, Madrid, Spain.

§Present address: Centro de Física Nuclear da Universidade de Lisboa, PT 1649-003 Lisbon, Portugal.

"Present address: TRIUMF, 4004 Wesbrook Mall, Vancouver, BC, V6T 2A3, Canada.

IPresent address: Dept. of Physics and Astronomy, University of Tennessee, Knoxville, Tennessee 37996, USA.

*** Present address: Dept. de Física Aplicada, Universidad de Salamanca, E-38005 Salamanca, Spain.
}

molecular dynamics (AMD) [5,6] and fermionic molecular dynamics (FMD) [7] calculations.

An important limitation for understanding the cluster structure of ${ }^{12} \mathrm{C}$ is the lack of a clear empirical picture of the existence of cluster states in ${ }^{12} \mathrm{C}$ above the $0^{+}$state at $7.65 \mathrm{MeV}$. As an example, AMD calculations predict $2^{+}, 3^{-}, 4^{+}, 5^{-}$, $6^{+}$states within the first $10 \mathrm{MeV}$ above the $3 \alpha$ threshold, which have not been identified to date [6]. The experimental challenge is that states with a well-developed cluster structure couple strongly to the $3 \alpha$ final state and therefore they are expected to quickly acquire large decay widths of the order of a MeV or more. Such resonances are hard to identify in most experimental approaches since they will overlap with each other and with other already known states in the same energy region.

Despite this difficulty there has recently been important progress. From intermediate energy scattering reactions induced by either $\alpha$ particles [8] or protons [9,10], evidence for a $2^{+}$resonance around $9-10 \mathrm{MeV}$ in ${ }^{12} \mathrm{C}$ has emerged (see also the discussion in Ref. [11]). The $2^{+}$resonance is found below the much more strongly populated $3^{-}$state at 9.64 $\mathrm{MeV}$. The same energy region is probed by $\beta$-decay studies of ${ }^{12} \mathrm{~N}$ and ${ }^{12} \mathrm{~B}$ [12-16], where overlapping $0^{+}$and $2^{+}$ resonances are found around $11 \mathrm{MeV}$. It is not yet clear why the two approaches give somewhat different results. From the reactions ${ }^{12} \mathrm{C}(\alpha, 3 \alpha) \alpha$ and ${ }^{9} \mathrm{Be}(\alpha, 3 \alpha) n$ there is also evidence for a new $4^{+}$resonance $800 \mathrm{keV}$ below the known $4^{+}$resonance at $14.08 \mathrm{MeV}$ [17].

To facilitate the identification of the new $2^{+}$and $4^{+}$ resonances, and the search for other new resonances, the energies, widths, and decay modes of the already known resonances must be accurately determined. The natural parity states can $\alpha$ decay both to the $0^{+}$ground state and the $2^{+}$first excited state of ${ }^{8} \mathrm{Be}$, while for the unnatural parity states only the latter decay channel is allowed. Due to the large width of the 
$2^{+}$state in ${ }^{8} \mathrm{Be}(\Gamma=1.5 \mathrm{MeV})$, the $\alpha$ spectrum of this decay branch is strongly influenced by interference effects, which has been the subject of several dedicated studies [18-20]. Thus, to fully characterize the $\alpha$ decay of unnatural parity states in ${ }^{12} \mathrm{C}$, the $3 \alpha$ momentum distribution of the ${ }^{8} \mathrm{Be} 2^{+}$branch must be measured, while for the natural-parity states the branching ratios for the two ${ }^{8} \mathrm{Be}$ decay branches are also required.

The present paper reports on experimental results pertaining to this goal obtained from the measurement of the two reactions ${ }^{10} \mathrm{~B}\left({ }^{3} \mathrm{He}, p \alpha \alpha \alpha\right)$ and ${ }^{11} \mathrm{~B}\left({ }^{3} \mathrm{He}, d \alpha \alpha \alpha\right)$ in complete kinematics. Here, we report on precision measurements of the properties of the $9.64\left(3^{-}\right), 10.81\left(1^{-}\right), 11.83\left(2^{-}\right), 13.35\left(4^{-}\right), 14.08\left(4^{+}\right)$, $16.11\left(2^{+}\right)$, and $20.5 \mathrm{MeV}\left(3^{+}\right)$resonances. Several results from the same experiment have already been published: the decay mechanism of the unnatural-parity states at $11.83\left(2^{-}\right)$, $12.71\left(1^{+}\right)$, and $13.35 \mathrm{MeV}\left(4^{-}\right)[21]$, the ${ }^{8} \mathrm{Be}(\mathrm{g} . \mathrm{s}$.) branching ratio of the $7.65 \mathrm{MeV}$ state [22], and the $\gamma$ decay of the 12.71 and $15.11 \mathrm{MeV}\left(1^{+}\right)$states to unbound states [19].

Section II describes the experiment and the analysis procedure. Due to the large amount of unwanted background channels, great care was taken to separate out the channels of interest. Section III discusses the experimental results and compares them with the results of previous experimental studies as well as various theoretical model predictions. A subsection of Sec. III is dedicated to the search for the proposed resonances observed in recent experimental studies. Finally, Sec. IV summarizes the paper.

\section{EXPERIMENT AND ANALYSIS}

The ${ }^{10} \mathrm{~B}\left({ }^{3} \mathrm{He}, p \alpha \alpha \alpha\right)$ reaction at a beam energy of $4.9 \mathrm{MeV}$ and the ${ }^{11} \mathrm{~B}\left({ }^{3} \mathrm{He}, d \alpha \alpha \alpha\right)$ reaction at a beam energy of $8.5 \mathrm{MeV}$ were studied at the Centro de Microanálisis de Materiales accelerator facility located in Madrid, Spain. The setup consisted of four double-sided silicon strip detectors (DSSSDs) $60 \mu \mathrm{m}$ thick [23], each backed by a thick silicon pad $1.5 \mathrm{~mm}$ thick used for particle identification and to fully stop the high energy protons and deuterons from the two reactions. One DSSSD has 32 vertical $\times 32$ horizontal strips of $2 \mathrm{~mm}$ width, while the other three DSSSDs have 16 vertical $\times 16$ horizontal strips of $3 \mathrm{~mm}$ width. The detectors were located $4 \mathrm{~cm}$ from the target, giving a total solid-angle coverage of $38 \%$ of $4 \pi$. At this distance, the angular resolution of the $32 \times 32$ DSSSD pixel is $2^{\circ}$ while the resolution of the $16 \times 16$ DSSSD pixel is $3^{\circ}$. The large solid angle combined with the high segmentation of the DSSSDs gives complete kinematics information on the triple- $\alpha$ breakup of populated resonances in ${ }^{12} \mathrm{C}$. A detailed description of the setup and of the analysis techniques is given in Ref. [24] (see also the already mentioned papers [19,21,22]).

The ${ }^{12} \mathrm{C}$ excitation energy is determined from the momentum of the outgoing light ion, i.e., the proton in the reaction with the ${ }^{10} \mathrm{~B}$ target and the deuteron in the reaction with the ${ }^{11} \mathrm{~B}$ target. The complete kinematics information allows the removal of most random coincidences, and the main challenge is therefore contributions to the $p \alpha \alpha \alpha$ and $d \alpha \alpha \alpha$ final states from reaction channels that do not involve the formation of a ${ }^{12} \mathrm{C}$ resonance as discussed in detail below.

\section{A. Analysis procedure}

The goal of the analysis is to determine energies, widths, and partial decay branches for selected resonances in ${ }^{12} \mathrm{C}$. We restrict the analysis to complete kinematics events, which means that either two or three $\alpha$ particles are detected in coincidence with a proton or deuteron. If only two $\alpha$ particles are detected, the momentum of the missing particle is determined by energy and momentum conservation.

The energy spectra were calibrated internally using wellknown states whose energies were taken from Ref. [25]. The ground state and the 4438.91(31), 9641(5), and 12710(6) keV states were used for the ${ }^{11} \mathrm{~B}$ target data, while for the ${ }^{10} \mathrm{~B}$ target data, the 9641(5), 12710(6), and 16105.8(7) keV states were used. This procedure resulted in a $5 \mathrm{keV}$ systematic uncertainty in the energy determination, and naturally prevents us from providing new information on the energy of the resonances used for the calibration. The energies and widths of resonances were determined by assuming Breit-Wigner peak shapes convoluted with a Gaussian experimental resolution on top of a smooth background to account for competing reaction channels and possible broad, hitherto unidentified, resonances in ${ }^{12} \mathrm{C}$. This procedure is justified because the resonances discussed here have relatively small widths and because we are insensitive to the detailed behaviors of the tails of the resonances, making a full $R$-matrix analysis unnecessary. The excitation-energy resolution is determined by the intrinsic detector resolution, the angular resolution, and variations in the energy loss in the target and the detector dead layer, so that there is a dependency on the energy and angle of the primary ejectile, and on the detector. The excitation-energy resolution was $60-120 \mathrm{keV}$ for the ${ }^{11} \mathrm{~B}$ target and $55-85 \mathrm{keV}$ for the ${ }^{10} \mathrm{~B}$ target.

The systematic errors of the widths were dominated by the treatment of the background. The errors were estimated individually for each resonance using different assumptions for the background (either first- or second-order polynomials) and by using different fit regions (wide and narrow). The possibility of interference with broad, hitherto unidentified, resonances in ${ }^{12} \mathrm{C}$, which could potentially distort the peak shape, was not considered. However, fit qualities were generally good, suggesting negligible distortion due to interference.

To determine the partial $\alpha$-decay branches, the states which decay sequentially via the ground state in ${ }^{8} \mathrm{Be}$ were separated using the invariant-mass technique. The ground state is situated $92 \mathrm{keV}$ above the $2 \alpha$ threshold, and the gate imposed on the $\alpha-\alpha$ relative energy was between 0 and $230 \mathrm{keV}$ in the case of multiplicity-three data, and between 40 and $150 \mathrm{keV}$ in the multiplicity-four data set.

\section{B. Background contribution}

As discussed above, the background contribution was the largest contributing factor to the uncertainty in the widths. The complete kinematics information allows the removal of essentially all random coincidences and decay channels that lead to multiparticle final states different from $p \alpha \alpha \alpha$ and $d \alpha \alpha \alpha$. It is, however, not possible to remove contributions from background channels that lead to the same multiparticle final states. Both reactions have contributing background 
channels; for the ${ }^{10} \mathrm{~B}$ target,

$$
{ }^{3} \mathrm{He}+{ }^{10} \mathrm{~B} \rightarrow\left\{\begin{array}{r}
p+{ }^{12} \mathrm{C} \rightarrow p+\alpha+{ }^{8} \mathrm{Be} \\
\alpha+{ }^{9} \mathrm{~B} \rightarrow \alpha+p+{ }^{8} \mathrm{Be} \\
\alpha+{ }^{9} \mathrm{~B} \rightarrow \alpha+\alpha+{ }^{5} \mathrm{Li} \\
{ }^{8} \mathrm{Be}+{ }^{5} \mathrm{Li} \rightarrow{ }^{8} \mathrm{Be}+p+\alpha
\end{array}\right\} \rightarrow p+3 \alpha,
$$

and for the ${ }^{11} \mathrm{~B}$ target,

$$
{ }^{3} \mathrm{He}+{ }^{11} \mathrm{~B} \rightarrow\left\{\begin{array}{c}
d+{ }^{12} \mathrm{C} \rightarrow d+\alpha+{ }^{8} \mathrm{Be} \\
\alpha+{ }^{10} \mathrm{~B} \rightarrow \alpha+d+{ }^{8} \mathrm{Be} \\
{ }^{8} \mathrm{Be}+{ }^{6} \mathrm{Li} \rightarrow{ }^{8} \mathrm{Be}+d+\alpha
\end{array}\right\} \rightarrow d+3 \alpha
$$

In addition, there may be contributions from direct reaction channels that do not involve the formation of intermediate resonances. Fortunately, it is possible to partly remove some of these channels. The $\alpha+{ }^{10} \mathrm{~B}$ channel from the ${ }^{11} \mathrm{~B}$ target has been the subject of a dedicated paper [26], and no narrow $(<0.5 \mathrm{MeV})$ resonances seem to interfere with the data analyzed here. Contributions from relatively narrow states in ${ }^{5} \mathrm{Li}$ and ${ }^{6} \mathrm{Li}$ are removed by simply placing gates on the corresponding peaks in $p$ - $\alpha$ and $d$ - $\alpha$ relative-energy spectra. These cuts will clearly affect the branching ratios and have therefore been included in the Monte Carlo simulation to determine detection efficiency (see Sec. III B). The contribution from the various background channels are best visualized by plotting $E_{\alpha_{i}}$ vs $E_{p / d}$ in the total center-of-mass reference frame. Figures 1 and 2 show such plots for the ${ }^{10} \mathrm{~B}$ and ${ }^{11} \mathrm{~B}$ target data, respectively. When fitting the ${ }^{12} \mathrm{C}$ excitation-energy projections, we model the background from other channels by using first- or second-degree polynomials.

\section{RESULTS AND DISCUSSION}

The final results are derived by averaging the values of the various data sets. This was done by summing the results from the multiplicity-three and -four data sets for each reaction, and then finally averaging together the results of both reactions in cases where the states were populated by both reactions. In all cases the results obtained from the different data sets were consistent with each other.

\section{A. Resonance energies and widths}

The final values for resonance energies and widths are given in Table I. Except for the 13.35 MeV state, the energies are within the error bars of the literature values, while the widths show substantial deviations. The literature value [25] of 13.352 (17) $\mathrm{MeV}$ for the energy of the $13.35 \mathrm{MeV}$ state is the weighted average of two values which are not consistent: 13.38(2) and 13.29(3) MeV. Therefore the deviation of our present value with the literature is not surprising. The width of the $9.64 \mathrm{MeV}\left(3^{-}\right)$state is larger than that given in the most recent $A=12$ compilation [25] by $\sim 1.4 \sigma$. However, our result agrees with the recent determination of $\Gamma=42(3)$ $\mathrm{keV}$ from measurements of ${ }^{12} \mathrm{C}\left(p, p^{\prime}\right)$ [9]. The width of the $10.84 \mathrm{MeV}\left(1^{-}\right)$resonance is below the literature value, while the width of the $13.35 \mathrm{MeV}\left(4^{-}\right)$resonance is larger than the literature value (see Table I). For the latter, as in the case of the resonance energy explained above, the literature value [25] is the average over several values that are not mutually
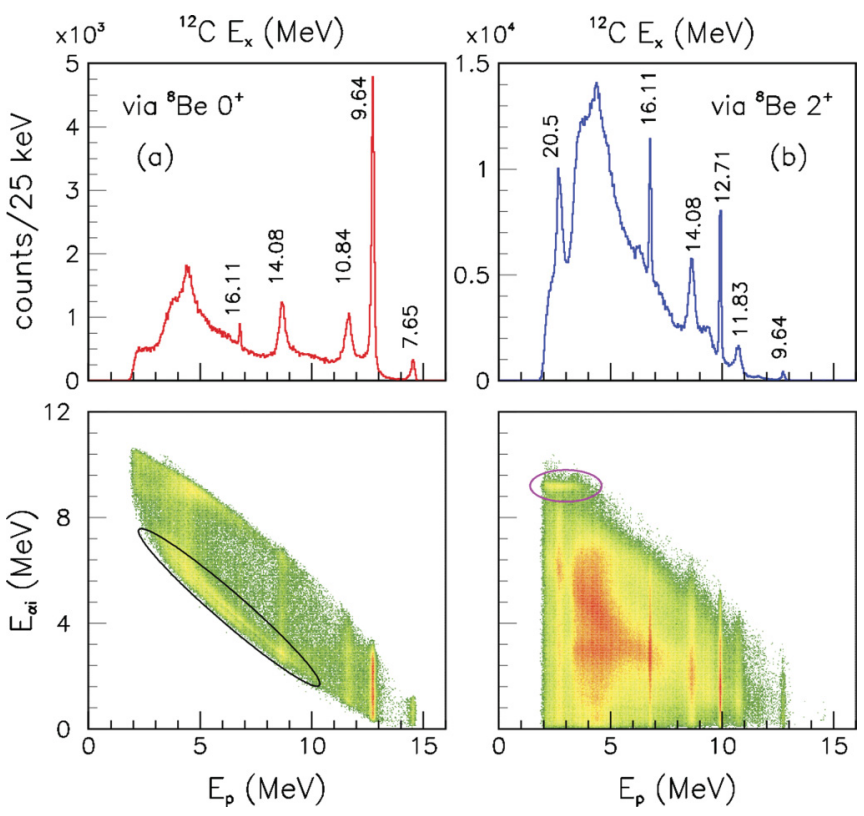

FIG. 1. (Color online) Multiplicity-four data from the ${ }^{10} \mathrm{~B}\left({ }^{3} \mathrm{He}, p \alpha \alpha \alpha\right)$ reaction. Individual $\alpha$ energies are plotted against proton energies in the c.m. frame. Excited states in ${ }^{12} \mathrm{C}$ appear as vertical bands of increased intensity in the two-dimensional spectrum, shown as peaks in the projections above the $E_{\alpha_{i}}$ vs $E_{p}$ plot figure. The data have been split into reactions that proceed via the ground state in ${ }^{8} \mathrm{Be}$ (a) and the rest (b), as explained in the text. The contribution from the ground state of ${ }^{5} \mathrm{Li}$ is circled in black in the lower part of (a). Similarly, the contribution from the narrow $2.36 \mathrm{MeV}$ state in ${ }^{9} \mathrm{~B}$ is circled in grey (pink) in (b). Both of these contributions have been removed before performing the fits of the resonances. There are clearly structures from broader states visible in both plots which cannot be fully removed.

consistent, and the disagreement is therefore not surprising: $500 \pm 80$ [27], $290 \pm 70$ [28], $430 \pm 100$ [29], $355 \pm 50$ [30], and $700 \pm 100$ [31] keV. Given the full kinematics approach resulting in much cleaner data, and the ability to clearly remove any ${ }^{8} \mathrm{Be}\left(0^{+}\right)$contribution, we feel the literature value for this width should be updated accordingly.

In addition to the states analyzed above, one can clearly see a peak at $\sim 18.5 \mathrm{MeV}$ excitation energy in both (a) and (b) of Fig. 1. Though this resonance seems to correspond to a $3^{-}$ state given in the literature at $18.6 \mathrm{MeV}$, we have chosen to not include this state in our analysis since there is a great deal of background from competing channels; see, e.g., the broad red region in Fig. 1(b) with apparent contributions from both the ${ }^{5} \mathrm{Li}$ and ${ }^{9} \mathrm{~B}$ channels.

In Table I we also compare the experimentally determined widths to theoretical values from a recent cluster-model calculation [32]. The theoretical widths are systematically a factor $\sim 2$ larger than the experimental widths, which indicates an $\alpha$ preformation factor of $\sim 0.5$.

\section{B. Partial $\alpha$-decay branches}

For the natural-parity resonances we extract the branching ratio to the ${ }^{8} \mathrm{Be}$ ground state and the ${ }^{8} \mathrm{Be} 2^{+}$state. There are two complications related to this observable. First, the 

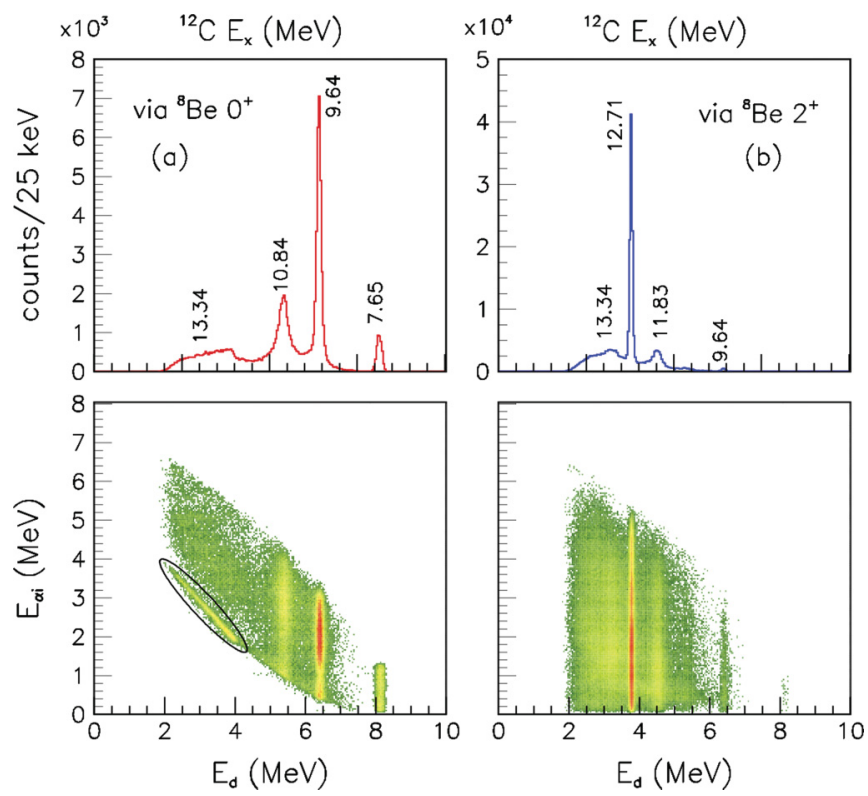

FIG. 2. (Color online) Same as Fig. 1, but for the ${ }^{11} \mathrm{~B}\left({ }^{3} \mathrm{He}, d \alpha \alpha \alpha\right)$ reaction. It is clear from the projections that the ${ }^{12} \mathrm{C}$ excitation energy spectrum is cleaner than that obtained with the ${ }^{10} \mathrm{~B}$ target. The contribution from the narrow $2.19 \mathrm{MeV}$ state in ${ }^{6} \mathrm{Li}$ is circled in black. Its contribution to the ${ }^{12} \mathrm{C}$ excitation-energy spectrum was removed before performing the fits.

experimental determination requires that the relative detection efficiencies for the two different $\alpha$-decay branches are reliably known. We determine these efficiencies by using Monte Carlo simulations taking into account the geometry of the detection setup, the kinematics of the reactions, and details of the data analysis. In the case of breakup through the $2^{+}$state in ${ }^{8} \mathrm{Be}$, the $3 \alpha$ breakup was simulated using-for simplicity - the CERNLIB routine GENBOD, which assumes a uniform population of the available phase space. The error introduced by not including angular correlations in the ${ }^{8} \mathrm{Be}\left(2^{+}\right)$ channel was estimated to be $5 \%$ by performing various Monte Carlo simulations using different realistic angular-correlation functions (see Ref. [24] for further details). In large part our insensitivity to angular correlations is due to the compact

TABLE I. Experimental values obtained for the energy and width of resonances in ${ }^{12} \mathrm{C}$. The literature values of $J^{\pi}, \Gamma$, and $E_{x}$ are taken from Ref. [25]. The $9.64 \mathrm{MeV}$ state was used for calibration, hence no energy is given for this state.

\begin{tabular}{lrcrrr}
\hline \hline $\begin{array}{l}E_{x} \text { of }{ }^{12} \mathrm{C} \\
(\mathrm{keV}) \text { lit. }\end{array}$ & $\begin{array}{c}\Gamma(\mathrm{keV}) \\
\text { lit. }\end{array}$ & $J^{\pi}$ & $\begin{array}{c}E_{x}(\mathrm{keV}) \\
\text { present }\end{array}$ & $\begin{array}{r}\Gamma(\mathrm{keV}) \\
\text { present }\end{array}$ & $\begin{array}{r}\Gamma(\mathrm{keV}) \\
\text { theory }\end{array}$ \\
\hline $9641(5)$ & $34(5)$ & $3^{-}$ & & $43(4)$ & 68 \\
$10844(16)$ & $315(25)$ & $1^{-}$ & $10847(4)$ & $272(5)$ & 475 \\
$11828(16)$ & $260(25)$ & $2^{-}$ & $11837(4)$ & $229(8)$ & 452 \\
$13352(17)$ & $375(40)$ & $4^{-\mathrm{b}}$ & $13305(9)$ & $510(40)$ & 1035 \\
$14083(15)$ & $258(15)$ & $4^{+}$ & $14078(5)$ & $273(5)$ & 606 \\
$20500(100)$ & $300(50)$ & $\left(3^{+}\right)$ & $20553(5)$ & $245(7)$ & \\
\hline
\end{tabular}

${ }^{\mathrm{a} C l u s t e r ~ m o d e l ~[32] . ~}$

${ }^{\mathrm{b}}$ The previous $J^{\pi}=2^{-}$assignment has been revised in two recent articles $[21,33]$.
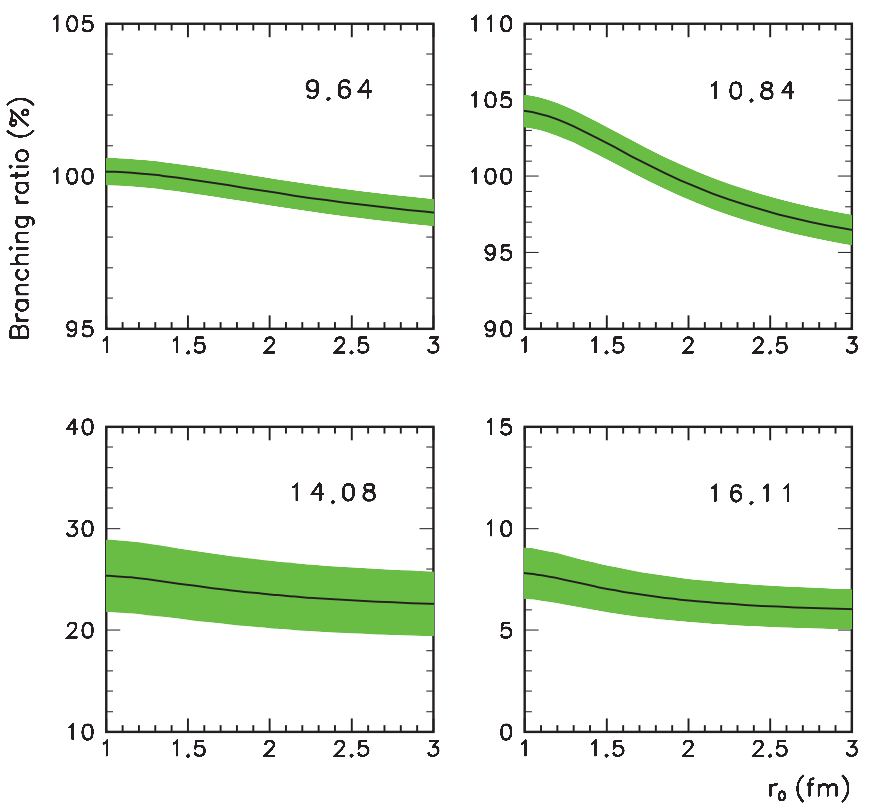

FIG. 3. (Color online) Ground-state branching ratios vs $r_{0}$. The green-shaded bands indicate a $1 \sigma$ uncertainty obtained by adding the systematic and statistical uncertainties in quadrature. A value of $r_{0}=1.41 \mathrm{fm}$ was used to determine the branching ratios.

detector setup designed to cover a large part of the phase space. Second, from a theoretical point of view, the ${ }^{8} \mathrm{Be}$ ground-state branch is not restricted to the $92 \mathrm{keV}$ peak in the $\alpha-\alpha$ relative energy spectrum. This is due to the proximity of the ${ }^{8} \mathrm{Be}$ ground state to the $\alpha-\alpha$ threshold, which causes the high energy tail of the resonance to blow up and create what is called a "ghost" [34]. We estimate this effect by using the $R$-matrix formalism [35]. The choice of exit- and entrancechannel radii can have a significant impact on the relative contribution of the ghost and, hence, on the deduced branching ratios. Increasing the exit-channel radius has the effect of diminishing the contribution from the ghost, which in turn will decrease the deduced ground-state branching ratio. This effect is illustrated in Fig. 3, where the ground-state branching ratios are plotted vs increasing exit-channel radius. The partial branches given in Table II used the typical reduced radius of $r_{0}=1.41 \mathrm{fm}$, giving an exit-channel radius of $a_{c}=r_{0}\left(4^{1 / 3}+\right.$ $\left.4^{1 / 3}\right)=4.5 \mathrm{fm}$ which corresponds to the best-fit found in Ref. [36].

Table II shows the deduced branching ratios $\Gamma_{\alpha_{0}} / \Gamma$ to the ground state of ${ }^{8} \mathrm{Be}$ with and without corrections due to the ghost effect. One can see the importance of including this effect, e.g., for the 9.64 and $10.84 \mathrm{MeV}$ resonances where the fraction of $\alpha$ decays bypassing the ${ }^{8} \mathrm{Be}$ ground-state peak is consistent with the expected ghost contribution, and therefore there is no need to invoke other decay branches for these states. The experimental branching ratios given in the literature are not corrected for the ghost effect, and they should therefore not be compared to the corrected values, which are mostly of theoretical interest.

The theoretical branching ratios given in Table II are the result of cluster-model calculations [37] which use the complex scaled hyperspherical adiabatic expansion mentioned. The 
TABLE II. Branching ratios of natural-parity resonances in ${ }^{12} \mathrm{C}$ decaying to the ground state of ${ }^{8} \mathrm{Be}$. The branching ratios are shown with and without correcting for the decay via the ghost of the ${ }^{8} \mathrm{Be}$ ground state. Note that the branching ratios are calculated assuming the typical reduced radius of $r_{0}=1.41 \mathrm{fm}$. The result for the $\alpha$ decay of the $7.65 \mathrm{MeV}\left(0^{+}\right)$state is discussed separately in Ref. [22], and included in this table for completeness.

\begin{tabular}{lccccc}
\hline \hline $\begin{array}{l}E_{x}{ }^{12} \mathrm{C} \\
(\mathrm{MeV})\end{array}$ & $\mathrm{J}^{\pi}$ & $\begin{array}{c}\Gamma_{\alpha_{0}} / \Gamma \% \\
\text { literature }\end{array}$ & $\begin{array}{c}\Gamma_{\alpha_{0}} / \Gamma \% \\
\text { no corr. }\end{array}$ & $\begin{array}{c}\Gamma_{\alpha_{0}} / \Gamma \% \\
\text { corr. }\end{array}$ & $\begin{array}{c}\Gamma_{\alpha_{0}} / \Gamma \% \\
\text { cluster [37] }\end{array}$ \\
\hline 7.65 & $0^{+}$ & $>99.5[22,38]$ & & $100.00(1)$ & 95 \\
9.64 & $3^{-}$ & $97.2[33]$ & $98.0(4)$ & $100.0(4)$ & 96 \\
10.84 & $1^{-}$ & & $94.3(9)$ & $102.6(9)$ & 70 \\
14.08 & $4^{+}$ & $17(4)[33,39]$ & $22(3)$ & $25(3)$ & 20 \\
16.11 & $2^{+}$ & $4.4(8)^{\mathrm{a}}$ & $5.8(9)$ & $7.2(9)$ & \\
\hline \hline
\end{tabular}

${ }^{\mathrm{a}}$ Ref. [25] gives $\Gamma_{\alpha_{0}}=0.290(45) \mathrm{keV}$ and $\Gamma_{\alpha_{1}}=6.3(5) \mathrm{keV}$.

16.11 MeV resonance has isospin $T=1$ and is therefore not reproduced by the cluster model. However, the cluster model does predict a $2^{+}$resonance at $13.8 \mathrm{MeV}$ with a ground-state branching ratio of $4 \%$. The cluster model does a good job at reproducing the branching ratios except for the $10.84 \mathrm{MeV}$ resonance. Our results could be used to improve the parameters used in the model.

The partial widths to the ground state of ${ }^{8} \mathrm{Be}, \Gamma_{\alpha_{0}}$, are given in Table III and are compared with the predictions of various cluster models. The partial width of a resonance gives an indication of the preformation probability of the $\alpha$ cluster, and is given by $\Gamma=2 P_{l} \gamma_{l}^{2}$, and the dimensionless reduced width is given by

$$
\theta_{\alpha}^{2}=\gamma_{l}^{2} / \gamma_{W}^{2},
$$

where the Wigner limit $\gamma_{W}^{2}$,

$$
\gamma_{W}^{2}=\frac{3}{2} \hbar^{2} / 2 \mu a_{c}^{2},
$$

represents the maximum width assuming a preformed $\alpha$ cluster. We use the same radius $a_{c}$, as discussed above, so that the errors only reflect the uncertainty in the width and not the uncertainty in the choice of the radius.

The dimensionless reduced width of the $9.64 \mathrm{MeV}$ resonance for the ${ }^{8} \mathrm{Be}\left(0^{+}\right)$channel is determined to be 0.75 , which is quite large compared to the other resonances. This is expected since the branching ratio to the ground state is $100 \%$ and indicates that this $3^{-}$resonance is highly clustered. However, the calculations of Ref. [42] gives $\theta_{\alpha_{0}}^{2}=0.165$ (GCM calculation) and 0.171 (RGM calculation) for a radius of $a_{c}=5 \mathrm{fm}$ for the $3^{-}$state. If we choose a channel radius of $a_{c}=5 \mathrm{fm}$, we obtain $\theta_{\alpha_{0}}^{2}=0.35(3)$, only a factor of 2 larger. The reduced width of the $16.11 \mathrm{MeV}$ resonance is negligible, which is expected due to its $T=1$ nature. Finally, the fairly low value of $\theta_{\alpha_{0}}^{2}$ for the 10.84 and $14.08 \mathrm{MeV}$ states indicates only a small level of clustering, which explains why these states are well described by the shell model [43].

\section{Evidence for new resonances}

As discussed in the Introduction, experimental evidence for new $2^{+}$and $4^{+}$resonances has recently emerged [8-17]. In this section, we briefly discuss signatures of these resonances in the present data set. In Figs. 1 and 2 the ${ }^{12} \mathrm{C}$ excitation spectra are shown, separated according to the decay modes through the ${ }^{8} \mathrm{Be}$ ground state and other intermediate states. In the ${ }^{8} \mathrm{Be}$ ground-state channel, there is additional strength in the energy region of $9-10 \mathrm{MeV}$, which is not accounted for by the "known" resonances, i.e., the ones listed in Table I. The known broad $0^{+}$state in the $10-11 \mathrm{MeV}$ region is likely to account for a significant part of this additional strength, the shape of which will differ from a simple Breit-Wigner profile due to penetrability effects and interference with the $7.65 \mathrm{MeV}$ state. In addition, the $9.64 \mathrm{MeV}\left(3^{-}\right)$resonance strongly dominates this region of excitation energy. Our analysis of this region is further hindered by possible contributions from broad states in the background channels (e.g., the ${ }^{6} \mathrm{Li}$ channel). In conclusion, it is not possible to claim evidence for a new $2^{+}$resonance around $9-10 \mathrm{MeV}$ in the present data.

Regarding the postulated $4^{+}$resonance situated at 13.3 MeV, we see evidence for a broad structure in the $13 \mathrm{MeV}$ region in Fig. 2 (left). The intensity in this region cannot be accounted for by the $13.35 \mathrm{MeV}\left(4^{-}\right)$resonance since decay via the ground state of ${ }^{8} \mathrm{Be}$ is restricted to natural-parity states. Unfortunately, this region has significant background from the ${ }^{6} \mathrm{Li}$ channel and gets more complicated by the presence

TABLE III. A comparison of the partial widths of resonances in ${ }^{12} \mathrm{C}$ decaying to the ${ }^{8} \mathrm{Be}$ ground state with various theoretical models. The partial $\alpha$-decay widths are calculated from the new total widths given in Table I. The AMD model values are taken from Ref. [6] and use a value of $a_{c}=4 \mathrm{fm}$. There are also two cluster-model predictions in the table. Ref. [40] uses the microscopic $\alpha$-particle model of Bloch-Brink, taking into account the $3 \alpha$-cluster motion, while Ref. [41] uses a mean-field-type cluster potential. The reduced widths, $\theta_{\alpha_{0}}^{2}$, are calculated for the various states for $a_{c}=4.5 \mathrm{fm}$, so that the errors only reflect the uncertainty in the width and not the uncertainty in the choice of the radius.

\begin{tabular}{lcccccc}
\hline \hline $\begin{array}{l}E_{x}{ }^{12} \mathrm{C} \\
(\mathrm{MeV})\end{array}$ & $J^{\pi}$ & $\begin{array}{c}\Gamma_{\alpha_{0}}(\mathrm{keV}) \\
\text { present }\end{array}$ & $\begin{array}{c}\Gamma_{\alpha_{0}}(\mathrm{keV}) \\
\text { AMD [6] }\end{array}$ & $\begin{array}{c}\Gamma_{\alpha_{0}}(\mathrm{keV}) \\
\text { cluster [40] }\end{array}$ & $\begin{array}{c}\Gamma_{\alpha_{0}}(\mathrm{keV}) \\
\text { cluster [41] }\end{array}$ & $\begin{array}{c}\theta_{\alpha_{0}}^{2} \\
\text { present }\end{array}$ \\
\hline 7.65 & $0^{+}$ & & 0.04 & $7.7 \times 10^{-3}$ & $5.9 \times 10^{-3}$ & 0.19 \\
9.64 & $3^{-}$ & $43(4)$ & & 30 & 17 & $0.75(7)$ \\
10.84 & $1^{-}$ & $272(6)$ & & 340 & 158 & $0.16(1)$ \\
14.08 & $4^{+}$ & $68(8)$ & 40 & & $0.07(1)$ & $9.9(1.3) \times 10^{-5}$ \\
16.11 & $2^{+}$ & $0.38(5)^{\mathrm{a}}$ & & & 0.03 \\
\hline
\end{tabular}

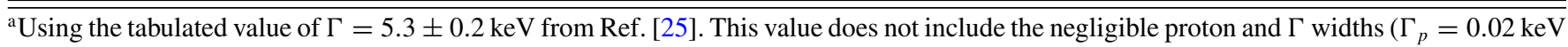
and $\left.\Gamma_{\gamma}=13 \mathrm{eV}\right)$. 
of broad $0^{+}$and $2^{+}$resonances in ${ }^{12} \mathrm{C}$ identified in $\beta$-decay studies [36]. Nevertheless, an attempt has been made to analyze the region between 12 and $13 \mathrm{MeV}$ through the use of Dalitz plots in a separate publication [44], and there is evidence for a broad resonance centered around $12.4 \mathrm{MeV}$ of unnatural parity with $J \geqslant 4$, i.e., the possibilities are $4^{-}, 5^{+}, 6^{-}, 7^{+}$, etc. An assignment of $4^{+}$ought to induce interference with the already known $4^{+}$resonance at $14.08 \mathrm{MeV}$, which we do not observe.

\section{CONCLUSION}

The resonance energies and widths in ${ }^{12} \mathrm{C}$ have been revisited in this paper from the study of both the ${ }^{10} \mathrm{~B}\left({ }^{3} \mathrm{He}, p \alpha \alpha \alpha\right)$ and ${ }^{11} \mathrm{~B}\left({ }^{3} \mathrm{He}, d \alpha \alpha \alpha\right)$ reactions measured in complete kinematics. Improved values for the widths, excitation energies, and decay channels are given for several established resonances. This complements already published results from the same experiment $[19,21,22,24]$. An attempt was made to search for new resonances which have been suggested in recent papers [8-11,17]. However, the background from other reaction channels did not allow us to either verify or refute the recently proposed new resonances.
The branching ratios to the ground state of ${ }^{8} \mathrm{Be}$ were extracted for the natural-parity states, and for the first time were corrected for the contribution of the ground-state ghost. The branching ratios, partial widths, and reduced partial widths were compared with theoretical predictions. It is clear from the comparisons that more work is needed from cluster-model calculations to fully describe the cluster structure of ${ }^{12} \mathrm{C}$. The properties of ${ }^{12} \mathrm{C}$ resonances determined in this work provide a challenge for theoretical models to reproduce them.

\section{ACKNOWLEDGMENTS}

We acknowledge support by the Spanish Research Grant FPA2009-07387 and Consolider Project CSD2007-00042, as well as support by the European Union Sixth Framework through RII3- EURONS/ JRA4-DLEP (Contract No. 506065). M.A. acknowledges the support of the CSIC I3P program cofinanced by the European Social Fund, and the support of the US Department of Energy, Office of Nuclear Physics, under Contract No. DE-AC02-06CH11357. O.S.K. acknowledges support from the Villum Kann Rasmussen Foundation.
[1] R. Roth, J. Langhammer, A. Calci, S. Binder, and P. Navrátil, Phys. Rev. Lett. 107, 072501 (2011).

[2] S. C. Pieper, Nucl. Phys. A 751, 516 (2005).

[3] E. Epelbaum, H. Krebs, D. Lee, and Ulf-G. Meißner, Phys. Rev. Lett. 106, 192501 (2011).

[4] M. Hjorth-Jensen, Physics 4, 38 (2011).

[5] Y. Kanada-En'yo, Phys. Rev. Lett. 81, 5291 (1998).

[6] Y. Kanada-En'yo, Prog. Theor. Phys. 117, 655 (2007).

[7] M. Chernykh, H. Feldmeier, T. Neff, P. von Neumann-Cosel, and A. Richter, Phys. Rev. Lett. 98, 032501 (2007).

[8] M. Itoh et al., Phys. Rev. C 84, 054308 (2011).

[9] M. Freer et al., Phys. Rev. C 80, 041303(R) (2009).

[10] W. R. Zimmerman, N. E. Destefano, M. Freer, M. Gai, and F. D. Smit, Phys. Rev. C 84, 027304 (2011).

[11] H. O. U. Fynbo and M. Freer, Physics 4, 94 (2011).

[12] H. O. U. Fynbo et al., Nature 433, 136 (2005).

[13] C. A. Diget et al., Nucl. Phys. A 760, 3 (2005).

[14] S. Hyldegaard et al., Phys. Lett. B 678, 459 (2009).

[15] S. Hyldegaard et al., Phys. Rev. C 80, 044304 (2009).

[16] C. A. Diget et al., Phys. Rev. C 80, 034316 (2009).

[17] M. Freer et al., Phys. Rev. C 83, 034314 (2011).

[18] H. O. U. Fynbo, Y. Prezado, U. C. Bergmann, M. J. G. Borge, P. Dendooven, W. X. Huang, J. Huikari, H. Jeppesen, P. Jones, B. Jonson, M. Meister, G. Nyman, K. Riisager, O. Tengblad, I. S. Vogelius, Y. Wang, L. Weissman, K. W. Rolander, and J. Äystö, Phys. Rev. Lett. 91, 082502 (2003).

[19] O. S. Kirsebom et al., Phys. Lett. B 680, 44 (2009).

[20] R. Alvarez-Rodríguez, H. O. U. Fynbo, A. S. Jensen, and E. Garrido, Phys. Rev. Lett. 100, 192501 (2008).

[21] O. S. Kirsebom et al., Phys. Rev. C 81, 064313 (2010).

[22] O. S. Kirsebom et al., Phys. Rev. Lett. 108, 202501 (2012).
[23] O. Tengblad et al., Nucl. Inst. Methods A 525, 458 (2004).

[24] M. Alcorta et al., Nucl. Inst. Methods A 605, 318 (2009).

[25] F. Ajzenberg-Selove, Nucl. Phys. A 506, 1 (1990).

[26] O. S. Kirsebom, M. Alcorta, M. J. G. Borge, M. Cubero, H. O. U. Fynbo, M. Madurga, and O. Tengblad, Phys. Rev. C 85, 054308 (2012).

[27] M. A. Reynolds, D. E. Rundquist, and R. M. Poichar, Phys. Rev. C 3, 442 (1971).

[28] M. A. Waggoner et al., Nucl. Phys. 88, 81 (1966).

[29] C. P. Browne, W. E. Dorenbusch, and J. R. Erskine, Phys. Rev. 125, 992 (1962).

[30] W. A. Schier and C. P. Browne, Phys. Rev. 138, B857 (1965).

[31] S. Hinds and R. Middleton, Proc. Phys. Soc. 78, 81 (1961).

[32] R. Alvarez-Rodríguez et al., Eur. Phys. J. A 31, 303 (2007).

[33] M. Freer et al., Phys. Rev. C 76, 034320 (2007).

[34] F. C. Barker and P. B. Treacy, Nucl. Phys. 38, 33 (1962).

[35] A. M. Lane and R. G. Thomas, Rev. Mod. Phys. 30, 257 (1958).

[36] S. Hyldegaard et al., Phys. Rev. C 81, 024303 (2010).

[37] R. Alvarez-Rodríguez, A. S. Jensen, E. Garrido, D. V. Fedorov, and H. O. U. Fynbo, Phys. Rev. C 77, 064305 (2008).

[38] J. Manfredi et al., Phys. Rev. C 85, 037603 (2012).

[39] D. D. Caussyn, G. L. Gentry, J. A. Liendo, N. R. Fletcher, and J. F. Mateja, Phys. Rev. C 43, 205 (1991).

[40] E. Uegaki et al., Prog. Theor. Phys. 62, 1621 (1979).

[41] J. Pei and F. Xu, Phys. Lett. B 650, 224 (2007).

[42] Y. Fujiwara et al., Suppl. Prog. Theor. Phys. 68, 29 (1980).

[43] P. Navrátil, V. G. Gueorguiev, J. P. Vary, W. E. Ormand, and A. Nogga, Phys. Rev. Lett. 99, 042501 (2007).

[44] O. S. Kirsebom, Few-Body Syst., doi: 10.1007/s00601-012-0441-z (2012). 